\title{
Editorial Expression of Concern: Topical Collection "Smart agriculture and geo-informatics"
}

Published online: 28 September 2021

(C) Saudi Society for Geosciences 2021

Following the publication of this topical collection of the Arabian Journal of Geosciences, the Publisher became aware of serious research integrity concerns within the collection. The peer review process was not carried out in accordance with the Publisher's peer review policy (see https://www. springer.com/gp/editorial-policies/peer-review-policyprocess), and may have been deliberately manipulated. All articles in this topical collection are currently being assessed. The Publisher will take further editorial action where appropriate, once this investigation is complete.

The authors were informed of the publication of this Editorial Expression of Concern using email addresses provided at submission. 